\title{
Engineering and Modifying Two-Dimensional Materials via Electron Beams
}

\author{
Xiaoxu Zhao ${ }^{1}$, Jiadong Dan ${ }^{1}$, Wu Zhou ${ }^{2}$, Kian Ping Loh $^{3}$, Stephen J. Pennycook ${ }^{1 *}$ \\ 1. Department of Materials Science and Engineering, National University of Singapore, 117575, \\ Singapore \\ 2. School of Physical Sciences and CAS Centre for Excellence in Topological Quantum Computation, \\ University of Chinese Academy of Sciences, Beijing 100049, China \\ 3. Department of Chemistry, National University of Singapore, Singapore, 117543, Singapore \\ * Corresponding author: steve.pennycook@nus.edu.sg
}

Electron beam (e-beam) irradiation damage is often regarded as a severe limitation to atomic scale study of two-dimensional (2D) materials using electron microscopy techniques. [1] However, energy transferred from the e-beam can also provide a way to modify $2 \mathrm{D}$ materials via defect engineering when the interaction of the beam with the sample is precisely controlled. [2-3]

Here, we successfully fabricate suspended monolayer Mo membranes from monolayer MoSe $\mathrm{films}_{2}$ ia selective e-beam ionization of Se atoms by scanning transmission electron microscopy (STEM). [4] The nucleation and subsequent growth of the Mo membranes are triggered by the formation and aggregation of Se vacancies as seen by atomic resolution sequential STEM imaging. Various novel structural defects and intriguing self-healing characteristics are unveiled during the growth. In addition, the monolayer Mo membrane is highly robust under the e-beam irradiation. Suspended monolayer metal membranes have never been prepared by conventional growth methods. It is likely that other metal membranes can be fabricated in a similar manner, and these pure metal-based 2D materials would remarkably diversify the category of 2D materials and may introduce profound novel physical properties.

In addition, we show that the migration of grain boundaries and healing of 2D planar defects in $\mathrm{MoSe}_{2}$ can also be triggered by an e-beam in a controllable manner. [5] By performing in-situ annealing experiments in an atomic-resolution scanning transmission electron microscope, we find that stacking faults and rotational disorder in multi-layered 2D crystals can be healed by grain boundary (GB) sliding, which works like a 'wiper blade' to correct all metastable phases into thermodynamically stable phase along its trace. The driving force for GB sliding is the gain in interlayer binding energy as the more stable phase grows at the expanse of the metastable ones. Density functional theory (DFT) calculations show that the correction of $2 \mathrm{D}$ stacking faults is triggered by the ejection of Mo atoms in mirror twin boundaries, followed by the collective migration of the 1D GB. Our study highlights the role of the often-neglected interlayer interactions for defect repair in 2D materials and shows that exploiting these interactions has significant potential for obtaining large-scale defect free 2D films. The atomically focused e-beam in a STEM can not only resolve the intrinsic atomic structure of materials with defects, but also provide new opportunities to modify the structure with subnanometer precision. [6]

\section{References:}

[1] H. P. Komsa et al., Physical Review Letters 109 (2012), p. 035503

[2] X. Zhao et al., MRS Bulletin 42 (2017), p. 667-676

[3] X. Zhao et al., Nano Letters 18 (2018), p. 482-490

[4] X. Zhao et al., Advanced Materials 30 (2018), p. 1707281 
[5] X. Zhao et al., Advanced Materials (accepted) (2019)

[6] The authors acknowledge funding from the National University of Singapore and Ministry of Education (MOE) for a Tier 2 grant "Atomic scale understanding and optimization of defects in 2D materials" (MOE2017-T2-2-139).
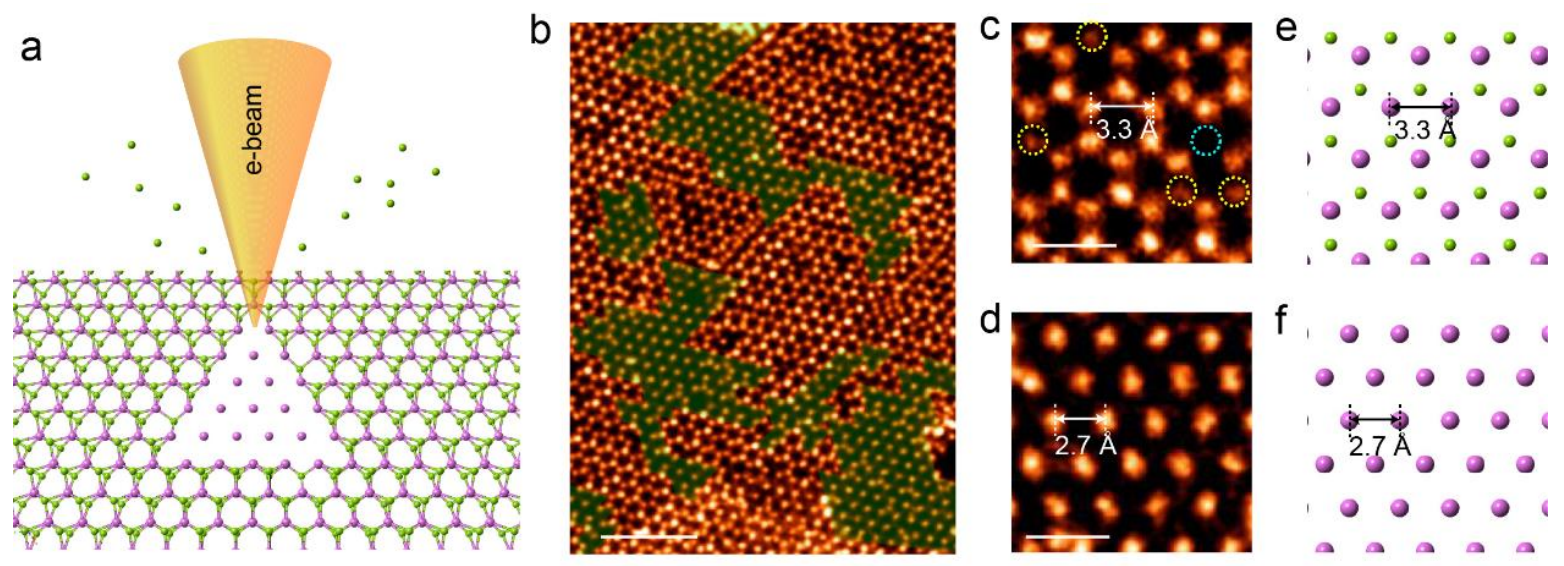

Figure 1. (a) Schematic illustration of the fabrication of a freestanding monolayer Mo membrane. (b) STEM-ADF image of as-fabricated Mo membranes embedded in a monolayer $\mathrm{MoSe}_{2}$ film. STEM-ADF images showing the monolayer $\mathrm{MoSe}_{2}$ film (c) and a monolayer Mo membrane (d). Corresponding atomic models are displayed in (e-f). Scale bars: $2 \mathrm{~nm}$ in (b), $0.5 \mathrm{~nm}$ in (c-d).
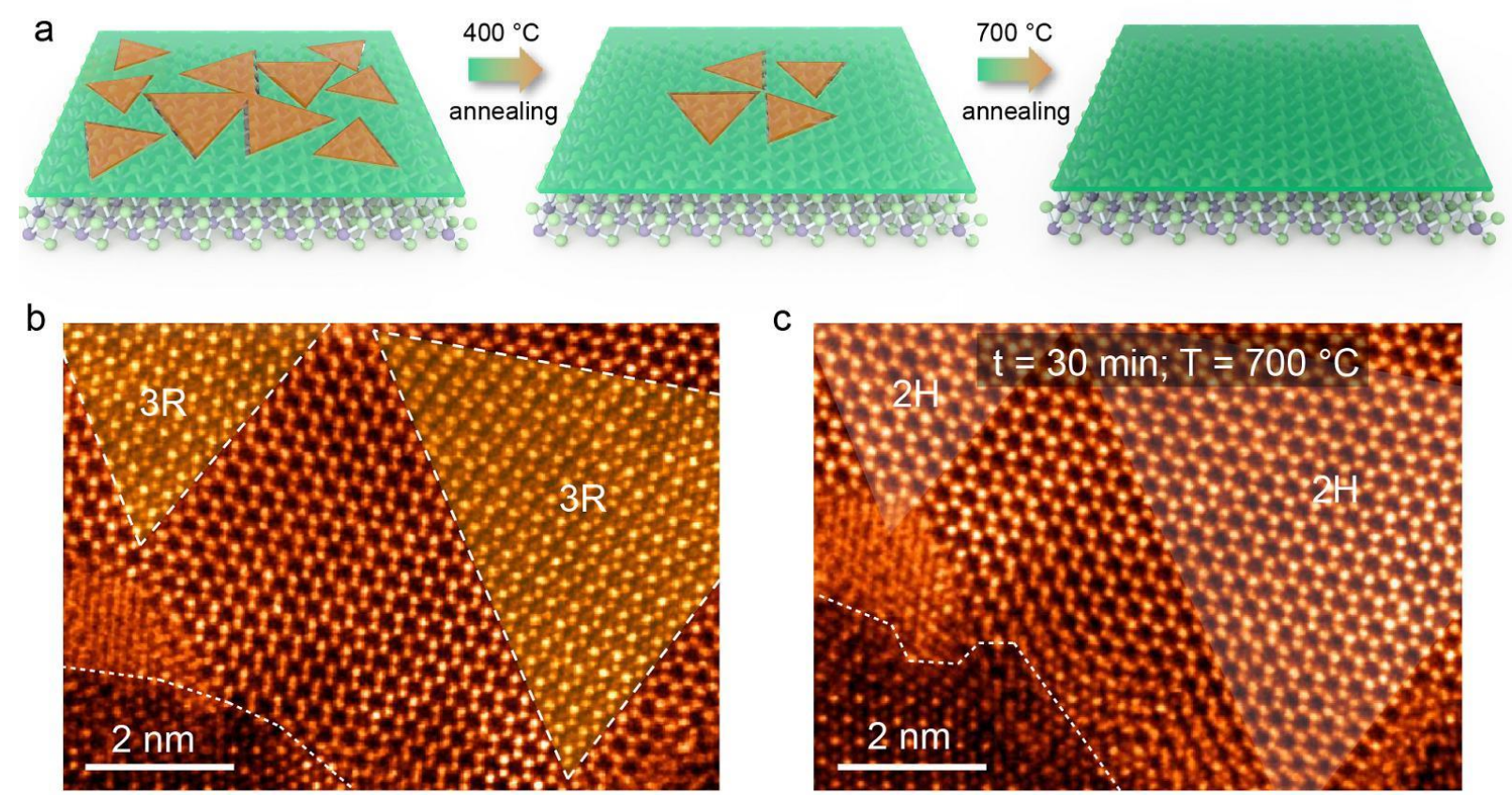

Figure 2. (a) Schematic illustration depicting the $\mathrm{MoSe}_{2}$ grain growth via GB migration and subsequent annihilation upon thermal annealing. Atomic-resolution STEM-ADF images of the same region of a bilayer $\mathrm{MoSe}_{2}$ film (a) before and (b) after in-situ heating at $700{ }^{\circ} \mathrm{C}$ for $30 \mathrm{~min}$. 